\title{
Erratum: Students' difficulties with vector calculus in electrodynamics [Phys. Rev. Phys. Educ. Res. 11, 020129 (2015)]
}

\author{
Laurens Bollen, Paul van Kampen, and Mieke De Cock
}

(Received 30 May 2017; published 13 June 2017)

DOI: 10.1103/PhysRevPhysEducRes.13.019905

This paper was published on 2 November 2015 with an incorrect reference. Reference [53] should be B. E. Hinrichs, Writing Position Vectors in 3-d Space: A Student Difficulty With Spherical Unit Vectors in Intermediate E\&M, AIP Conf. Proc. 1289, 173 (2010). 